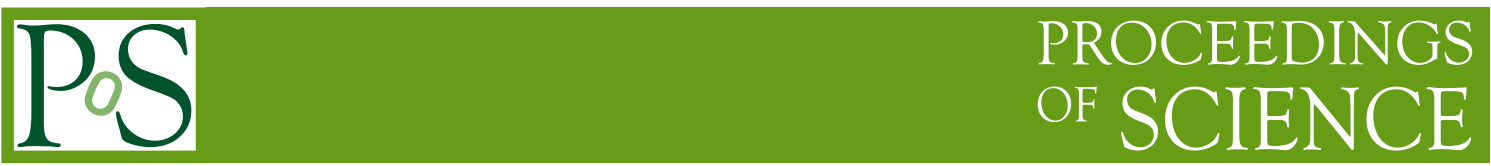

\title{
The ILD Software Tools and Detector Performance
}

\author{
Remi Ete ${ }^{a, *}$, on behalf of the ILD concept group \\ ${ }^{a}$ Deutsches Elektronen-Synchrotron, \\ Notkestraße 85, 22607 Hamburg, Germany \\ E-mail: remi.ete@desy.de
}

The ILD detector is designed for high precision physics at the ILC. It is optimized for particle flow event reconstruction with extremely precise tracking capabilities and highly granular calorimeters. Over the last decade, the ILD concept group has developed a suite of sophisticated software packages for simulation and reconstruction in the context of the iLCSoft ecosystem in collaboration with other future collider projects. We will present an overview of the ILD software from the detailed and realistic modeling of the detector with DD4hep, over the event reconstruction algorithms with their pattern recognition and particle flow algorithms to the high level reconstruction for flavor tagging and particle identification. Most of the these tools have been developed in a detector-agnostic way and are also applicable to other future collider experiments. Finally, we will present an overview of the resulting detector performance as shown in ILD's Interim Design Report (IDR) [1].

40th International Conference on High Energy physics - ICHEP2020

July 28 - August 6, 2020

Prague, Czech Republic (virtual meeting)

\footnotetext{
${ }^{*}$ Speaker
} 


\section{The iLCSoft stack}

The iLCSoft stack is a software ecosystem for collider experiments. While it has initially been developed for the LC experiments, it is also used by various other experiments and projects such as CEPC, CALICE, LCTPC [2] or EUTelescope [3] for simulation studies as well as for reconstruction and analysis of test beam data.

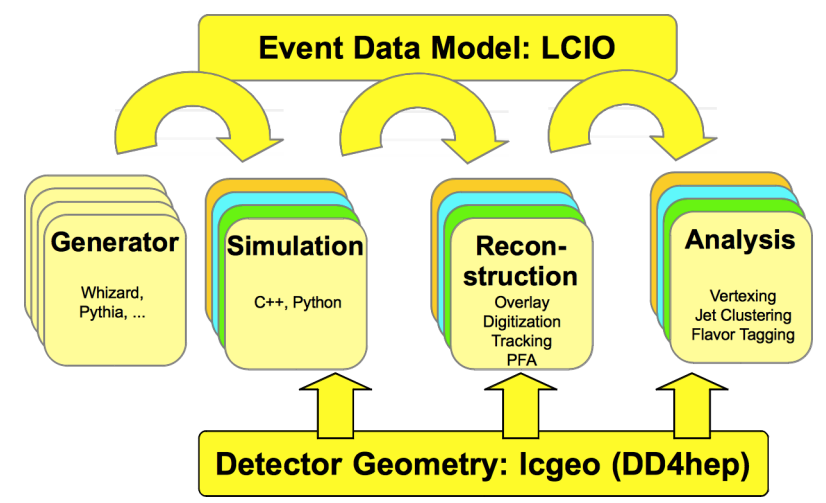

Figure 1: Overview of the iLCSoft components: the common event data model LCIO ensures coherent exchange of data between the all parts of the framework. DD4hep serves as unique source of geometry information.

The software stack comprises all necessary ingredients to perform detailed physics studies, as shown in Figure 1: event generators, responsible for generating particles at the collision point based on theoretical models; detector simulation, propagating these particles through the detectors; reconstruction algorithms, which form particle hypotheses out of the signals of the various subdetectors; and analysis software, to perform e.g. dedicated physics channel or detector optimization studies.

\subsection{The core packages}

The DD4hep [4] framework provides a generic detector description for all stages of a detector's life. DD4hep is based on the Geom package from R00T [5] and provides a unique source of geometry with multiple interfaces. Among the ones used in iLCSoft are DDG4, the gateway to full simulation with Geant4 [6] and DDRec, a high level view onto the geometry for reconstruction, in particular providing measurement surfaces and materials needed for track finding and fitting.

LCIO [7] is the event data model and format of the iLCSoft stack. It is one of the key units of this workflow, as it ensures a unique coherent format between processing steps, from generator to analysis. It is a standalone thread-safe IO library and data format, written in C++ with interfaces in Java, Fortran and Python. The format is binary (XDR) and the ZLIB algorithm is used for data compression. The versioning of data also makes the library backward compatible. A useful feature is the ability to link data objects via so-called LCRelation objects, making it possible to relate simulated and reconstructed objects and quickly navigate through them.

At the core of iLCSoft lies the Marlin [8] application framework. It is a light-weight HEP event processing framework based on LCIO, focused on reconstruction and analysis. Marlin 
defines a pipeline of ordered task units, so-called Processors, performing operations on LCIO data by reading, analysing and/or creating collections.

\subsection{The ILD detector}

A realistic model of the ILD [1] with dead material, gaps and imperfections is implemented in DD4hep. The ILD is a general-purpose HEP detector with close to $4 \pi$ coverage. Sub-detectors are placed in "onion-layers" from inside-out: a vertex detector (VTX), the silicon tracking detectors (SIT, FWD), a time project chamber for tracking (TPC), a silicon external tracking detector (SET), forward calorimeters (FCAL) in the endcap region, an electromagnetic calorimeter (ECal), a hadronic calorimeter calorimeter ( $\mathrm{HCal})$ and muon chambers.

Two variants have been implemented: ILD-L with the baseline TPC radius and ILD-S with a $20 \%$ smaller TPC radius. Figure 2 (left) shows hit maps in the r- $\phi$ view, combining one half of each detector model. For both variants, both ECal and HCal calorimeters are simulated with so-called hybrid options [1]. The layering of a sandwich calorimeter is generally composed of three different slice types: a.) the pure absorber part, b.) the sensitive part recording the signal and c.) additional slices such as e.g. electronics board and glue. Figure 2 (right) shows a simplified view of the HCal layering. The hybrid calorimeter option replaces the additional slices (c.) with the sensitive layer of the other technology, different from the first one, with similar radiation and interaction lengths. Using hybrid calorimeters allowed our simulation to run only once for all calorimeter options at the same time, saving CPU time and minimizing disk space. For reconstruction, one ECal and one HCal technology has to be chosen leading to four possible reconstruction models.
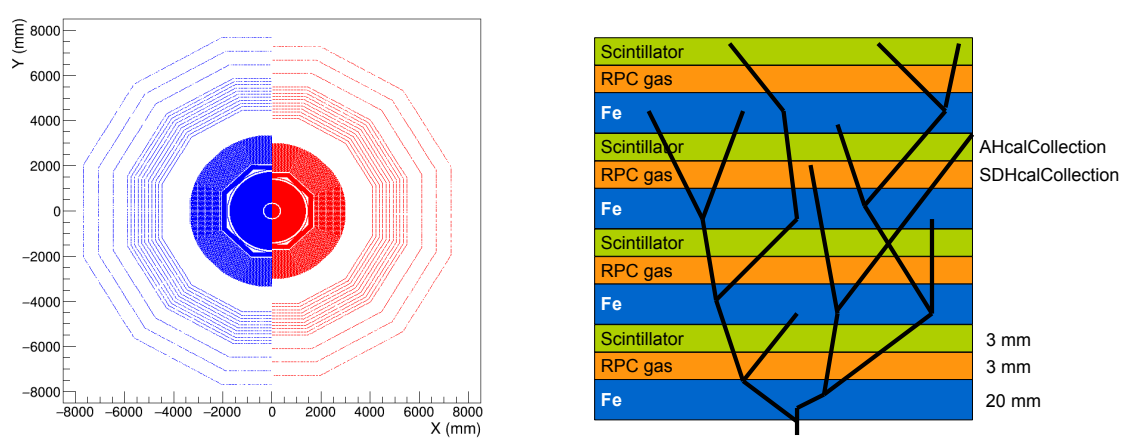

Figure 2: Left: side view of the two ILD models implemented in the simulation, ILD-L (blue) and ILD-S (red); right: simplified view of the hadronic calorimeter layer structure showing the hybrid sensitive parts.

\subsection{The ILD reconstruction}

The ILD reconstruction chain is implemented in the Marlin framework and consists of more than 60 reconstruction algorithms.

Background overlay During collisions, potential other background sources can be produced in parallel to the collision event, e.g. $\gamma \gamma$ to low $p_{t}$ hadrons or $e^{+} e^{-}$pairs. These background signals 
in the detector are overlaid onto the collision event for further processing. One bunch crossing is overlaid for the ILD event reconstruction.

Digitization The simulated hits in tracking and calorimeter detectors are then digitized. After applying an energy threshold, energy calibration constants are applied. For the calorimeters, additional effects such as inefficiency in electronics and noise randomization is also emulated, according to test beam results, to add more realism.

Track reconstruction The track reconstruction runs independently for each of the three different tracking regions, each producing a set of candidate tracks. For the VTX and SIT detectors, a bruteforce triplet seeding followed by a road search using extrapolations to the next layer is run, while for the FWD detectors, a cellular-automaton algorithm is applied. In the TPC detector a topological clustering in the outer TPC pad row layers for seeding is performed, followed by a Kalman-Filter based road search inwards.

The candidate tracks are then combined into a unique set of tracks, the remaining hits are merged into these tracks and a final track re-fitting is performed.

Particle flow The main event reconstruction is performed by PandoraPFA [9], that reconstructs individual particles candidates, called PFOs, out of reconstructed tracks and calorimeter hits. The fine granularity of calorimeters allows for a clear separation of energy deposits of individual particles during the clustering phase. Sophisticated pattern recognition algorithms are then used to identify clusters and associate each other to optimize the track-to-cluster compatibility for charged particles. An iterative re-clustering procedure recovers difficult situations of wrong clustering, e.g. when neutral particles make showers nearby a charged particle. Finally, a particle identification is performed to disentengle $e^{ \pm}, \gamma$, neutral hadron, $\mu^{ \pm}$and $\pi^{ \pm}$.

High-level reconstruction After identifying individual particles, additional reconstruction algorithms are run. Using the LCFIPIus [10] package, primary and secondary vertices are reconstructed. The particle identification is then performed using a Likelihood with variables such as the TPC measurement of $d E / d x$, shower shapes and PandoraPFA ID. $\pi^{0}$ and $\eta$ mesons are then identified by combining photon pairs. Finally, time of flight (TOF) estimates are calculated based on the ECal hit-times, assuming different time resolutions from 0 to $100 \mathrm{ps}$.

\section{The ILD detector performances}

Figure 3 (top-left) shows the momentum resolution obtained from single $\mu$-events at various polar angles and momenta. Central tracks, i.e. at $\theta=85$ and 40 degrees, reach the ILD design goal of an asymptotic transverse momentum resolution of $\sigma_{p t}=2.10^{-5} \mathrm{GeV}^{-1}$, indicated by the read line. The jet energy resolution is extracted using $u d s$ di-jet events generated at fixed center-of-mass energies, without ISR and beam energy spectrum. The jet energy resolution is calculated using $E_{j e t}=E_{t o t} / 2$ as:

$$
\frac{\sigma_{E_{j e t}}}{E_{j e t}}:=\frac{r m s_{90}\left(E_{j e t}\right)}{\text { mean }_{90}\left(E_{j e t}\right)}
$$



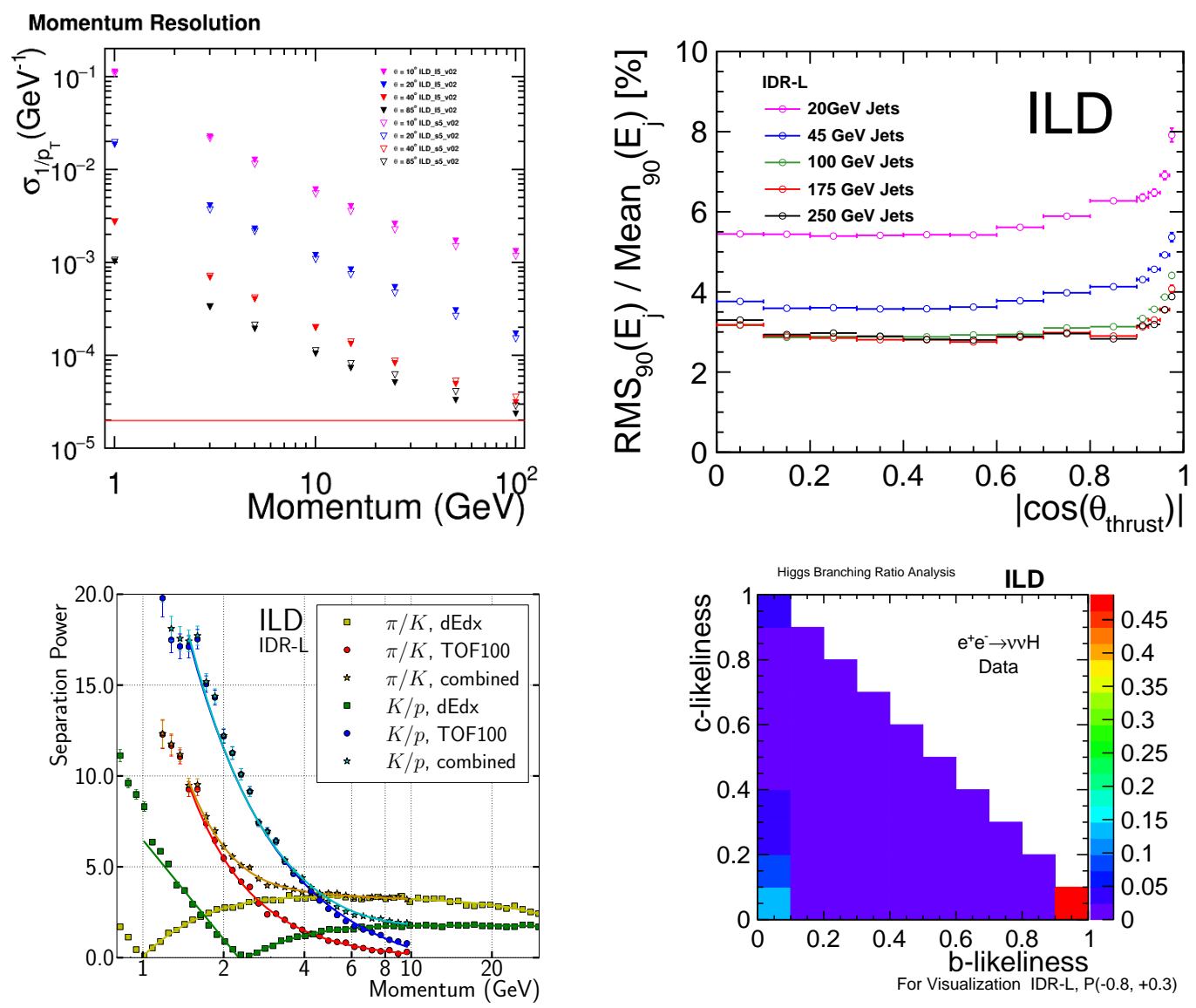

Figure 3: ILD detector performances. Top left: momentum resolution as a function of momentum; top right: jet energy resolution as a function of $\cos \theta$; bottom left: $\pi / K$ and $K / p$ separation power using TPC $d E / d x$ combined with TOF from ECal; bottom right: $c$-likeliness vs. $b$-likeliness for $H \rightarrow b \bar{b}$ using the LCFIPlus algorithm.

where mean $_{90}$ and $r m s_{90}$ are respectively the mean value and the rms of the central $90 \%$ of the jet energy distribution. A value of 3-4 \% is reached in the barrel and endcap regions for the two detector variants and on a broad energy range as shown on Figure 3 (top-right). The ILD TPC can measure the energy loss $d E / d x$ which can be used to separate charged particle species. Additionally, we have been investigating the use of TOF measurements using the first layers of the ECal. Figure 3 (bottom-left) shows the separation power for $\pi / K$ and $K / p$ particle species using $d E / d x$, TOF and both combined. The use of TOF at low momentum improves the separation power when combined with $d E / d x$, in particular in the region for those momenta where the $d E / d x$ bands of different particle species cross.

The identification of $b$ and $c$ jets in collisions is particularly important to study Higgs boson hadronic decays such as $e^{+} e^{-} \rightarrow v \bar{v} H$, with $H \rightarrow b \bar{b}$ or $H \rightarrow c \bar{c}$. Figure 3 (bottom-right) shows the $c$ - vs $b$-likeliness for $H \rightarrow b \bar{b}$. Almost all $H \rightarrow b \bar{b}$ events end up at the highest $b$-and lowest $c$-likeliness, indicating a very high $\mathrm{b}$ jet identification efficiency and purity. 


\section{Conclusion}

The iLCSoft is a highly successful suite providing all necessary tools to perform physics studies at linear colliders. Its recent additions comprise DD4hep, meanwhile adopted by many other experiments. iLCSoft is the basis of all detector and physics performance studies for the ILD detector. The state of the art detector and physics performances have been described into the ILD Interim Design Report. iLCSoft is evolving towards the usage of new technologies, with for example the introduction of multi-threading in the Marlin framework, and also by continuing the development of the high-level reconstruction tools.

\section{References}

[1] H. Abramowicz et al. [ILD Concept Group], "International Large Detector: Interim Design Report", arXiv:2003.01116 [physics.ins-det].

[2] J. Abernathy et al., "MarlinTPC: A Marlin based common TPC software framework for the LC-TPC collaboration". arXiv:0709.0790 [physics.ins-det].

[3] H. Perrey, "EUDAQ and EUTelescope: Software Frameworks for Test Beam Data Acquisition and Analysis". PoS TIPP2014 (2014), 353

[4] M. Frank, F. Gaede, M. Petric, \& A. Sailer. AIDASoft/DD4hep. Zenodo. http://doi.org/10.5281/zenodo.4066795

[5] R. Brun \& F. Rademakers, "ROOT - An Object Oriented Data Analysis Framework", Nucl. Instrum. Meth. 389 (1997), 81-86.

[6] S. Agostinelli et al., "GEANT4 - a simulation toolkit". Nucl. Instrum. Meth. A 506. (2003), 250-303

[7] F. Gaede, "LCIO - A persistency framework for linear collider simulation studies". arXiv:0306114 [physics.data-an].

[8] F. Gaede, "Marlin and LCCD—Software tools for the ILC". Nucl. Instrum. Meth. A 559 (2006), 177-180

[9] M. Thomson, "Particle Flow Calorimetry and the PandoraPFA Algorithm". Nucl. Instrum. Meth. A 611 (2009), 25-40

[10] T. Suehara \& T. Tanabe, "LCFIPlus: A Framework for Jet Analysis in Linear Collider Studies". Nucl. Instrum. Meth. A 808 (2016), 109-116 